\title{
Caso clínico: Síndrome de distrés respiratorio del adulto en paciente COVID-19 en ventilación mecánica con aspergilosis pulmonar invasiva
}

\author{
Clinical case: Adult respiratory distress syndrome in COVID-19 patient on \\ mechanical ventilation with invasive pulmonary aspergillosis
}

\author{
Juan Higuera Lucas',*, Diego Muñoz-Mingarro Molina \\ Medicina Intensiva, Hospital Universitario Ramón y Cajal, España.
}

Fecha de recepción: 30 de marzo de 2021 / Fecha de aceptación: 14 de mayo de 2021

\begin{abstract}
The bibliography on the management of the COVID-19 patient in intensive care units is increasing. Research and publication of results help to optimize the management of these patients and the consequent improvement of results. We present the case of a patient admitted to intensive care due to adult respiratory distress syndrome secondary to COVID-19 pneumonia and personal history of liver transplantation the previous year and pulmonary hypertension under treatment. During admission, the patient requires pronation, neuromuscular blockers, and nitric oxide. Invasive aspergillosis is diagnosed and requires percutaneous tracheostomy.
\end{abstract}

Key words: Intensive care, COVID-19, mechanical ventilation, ARDS.

\section{RESUMEN}

La bibliografía sobre el manejo del paciente COVID-19 en las unidades de cuidados intensivos va en aumento. La investigación y publicación de resultados ayudan a la optimización del manejo de estos pacientes y la mejora consecuente de resultados. Presentamos el caso de un paciente que ingresa en cuidados intensivos (UCI) por síndrome de distrés respiratorio del adulto secundario a neumonía COVID-19 y antecedentes de trasplante hepático el año previo e hipertensión pulmonar en tratamiento. Durante el ingreso, el paciente precisa pronación, relajación neuromuscular y óxido nítrico. Se diagnostica de aspergilosis invasiva y precisa traqueostomía percutánea.

Palabras clave: Medicina intensiva, COVID-19, ventilación mecánica, SDRA.

$\mathrm{D}$ esde hace más de un año, las unidades de cuidados intensivos han multiplicado sus ingresos por síndrome de distrés respiratorio del adulto (SDRA). Esto es debido a la pandemia producida por el coronavirus SARS-CoV-2 (COVID-19). Este virus, de probable origen animal, pertenece al grupo de los Rhinolophus, compartiendo el $96 \%$ de la secuencia genómica con algunos virus de este grupo[1]. Los primeros cuatro casos notificados de neumonía atípica datan del 26 de diciembre de 2019. El 7 de enero de 2020 se identificó el vi- rus. El 30 de enero, la Organización Mundial de la Salud (OMS) declaró a la enfermedad emergencia para la salud pública con preocupación internacional[2]. Un año después, esta enfermedad provoca más de 10.000 muertes al día en el mundo. Esto se debe a su alta capacidad de contagio, con un número reproductivo de 2,4, significa que, para contar con la conocida como inmunidad de rebaño, precisa estar inmunizada el $58 \%$ de la población[3].

El virus COVID-19 es un virus RNA que presenta una pro-

jhiguera.151@gmail.com

*ORCID: https://orcid.org/0000-0003-1778-2291 
teína S de membrana que se une a los receptores ACE2 de las células pulmonares inicialmente. Tras su entrada, se genera en el huésped una respuesta inmunológica alterada, con elevación desproporcionada de TNF alfa, $\| 1$, $\| 6$ y otros factores que contribuyen a la gravedad de la enfermedad, produciendo muchos casos de distrés respiratorio del adulto y neumonía grave[4].

Presentamos el caso de un paciente varón, 62 años, que es ingresado en Medicina Intensiva por esta patología. Hemos elegido este caso por presentar dos características que lo diferencian de otros. Los antecedentes personales de trasplante hepático y el diagnóstico de hipertensión pulmonar tratada farmacológicamente. Radiografía previa al ingreso (Figura 1).

\section{Antecedentes del paciente resumidos}

1. Trasplante hepático el día 31 de enero de 2020 por cirrosis hepática Child C 10 de etiología mixta enólica y por infección $\mathrm{VHC}$ naive + .

2. Estudio cardiológico previo a trasplante: Ecocardio (21 de enero de 2019). Ventrículo izquierdo no dilatado, levemente hipertrófico, con función sistólica normal. Disfunción diastólica tipo III. Elevación de las presiones de llenado. El ventrículo derecho moderadamente dilatado, con función sistólica normal. Aurícula derecha moderadamente dilatada. Insuficiencia tricúspidea moderada severa funcional que permite estimar una PSP > $70 \mathrm{mmHg}$ (HTP severa). Cateterismo cardiaco 17 de diciembre de 2019: hipertensión pulmonar moderada de predominio poscapilar (PAPs 59 $\mathrm{mmHg}$, PAPm 35 mmHg, PCP 24 mmHg, 1,7 UWood).

3. Estudios de función pulmonar ponen de manifiesto EPOC GOLD II grupo de riesgo B. FVC: 3510-92\%, FEV : 2270$76 \%$, FEV,/FVC 65\%.

4. Tratamiento domiciliario: omeprazol $20 \mathrm{mg}$, adiro $100 \mathrm{mg}$, macicentan $10 \mathrm{mg}$, tadalafilo $20 \mathrm{mg}$, advagraf $6 \mathrm{mg}$, certican $3 \mathrm{mg}$.

El paciente ingresa en el hospital el 22 de enero de 2021 por neumonía bilateral grave por COVID-19. Refiere diagnóstico de infección COVID-19 el día 18 de enero de 2021 mediante test de antígeno rápido al comenzar con fiebre, astenia y disnea sin otra clínica asociada. A las pocas horas de ingreso requerimiento de oxigenoterapia reservorio. Inicialmente buena mecánica respiratoria y saturación hasta deterioro clínico con aumento del trabajo respiratorio, motivo por el cual el 25 de enero de 2021 ingresa en Medicina Intensiva (Figura 2).

Se inicia soporte de oxigenación con oxigenoterapia de alto flujo, monitorizando respuesta mediante el índice de ROX[5], fracasando a las 2 horas. El paciente es intubado y conectado a ventilación mecánica. El tratamiento del COVID-19 ha sufrido cambios durante este año. En la actualidad sólo la dexametasona y recientemente el tacrolimus parecen tener un efecto favorable en cuanto a mortalidad[6]-[10]. El estudio RECOVERY, publicado en la revista New England Journal of Medicine, muestra mejoría en la supervivencia en los pacientes con neumonía y necesidad de oxígeno en los que se administra dexametasona. Así mismo ha demostrado menor mortalidad en pacientes en ventilación mecánica. Por el momento, los estudios no asocian su uso a mayor complicación o infección. Esto puede ser debido a la corrección de la disregulación inmune y trombótica[24].
Por esta razón, el paciente recibe tratamiento con dexametasona (6 mg) suspendiéndose su medicación inmunosupresora (tras cumplir ciclo de 10 días, se mantuvo hasta alcanzar nuevamente niveles de tacrolimus). Se inicia cobertura antibiótica empírica hasta descartar coinfección. En la actualidad, diversos estudios han demostrado una incidencia muy baja de coinfección bacteriana en la neumonía grave por coronavirus[11]. Tras descartar esta coinfección, la antibioterapia fue retirada (Figura 3). Se inició, además, profilaxis de úlcera de estrés con pantoprazol y enoxaparina profiláctica con 40 mg subcutánea.

Se realiza ecocardio a pie de cama. Se objetiva FEVI normal, con hipertrofia leve y ventrículo derecho no dilatado normofuncionante sin clara insuficiencia tricuspídea. Nuestro paciente se encontraba en tratamiento con antihipertensivos pulmonares previo a su ingreso en $\mathrm{UCI}$. Un artículo publicado en esta revista[12] habla de que $16,6 \%$ de los pacientes ingresados en $\mathrm{UCI}$

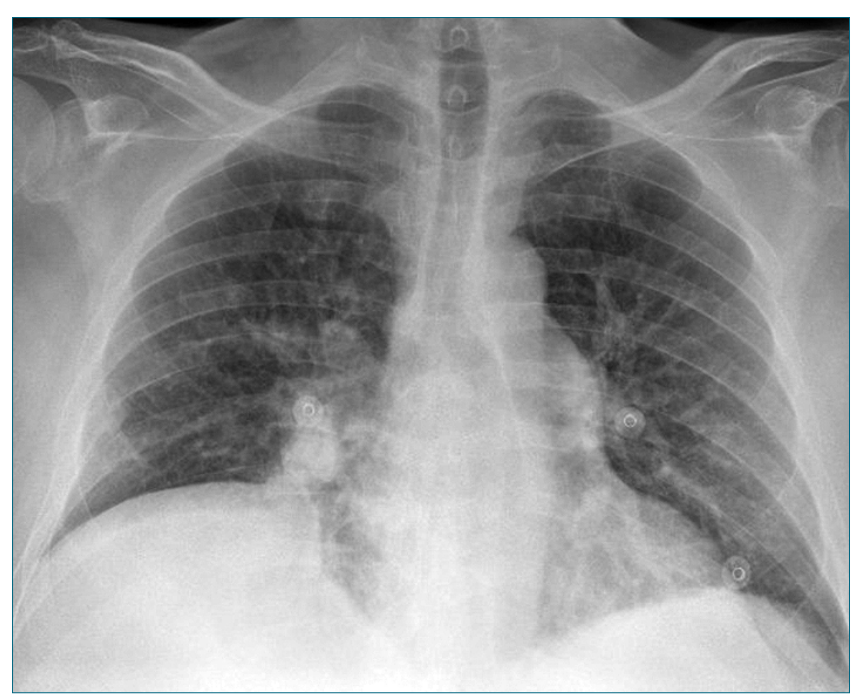

Figura 1. Radiografía del paciente previo al ingreso.

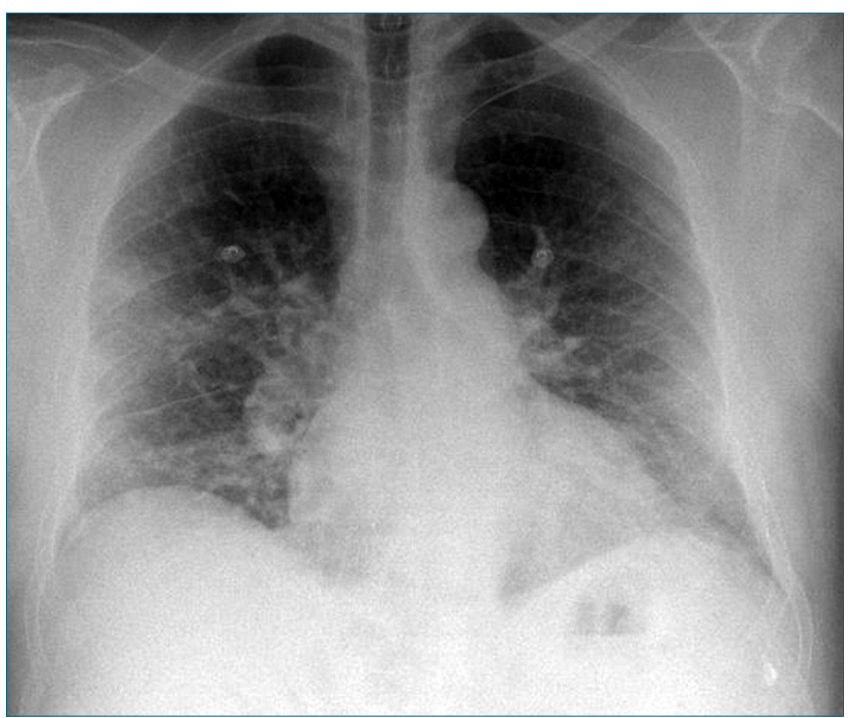

Figura 2. Radiografía del paciente el día de su ingreso hospitalario. 


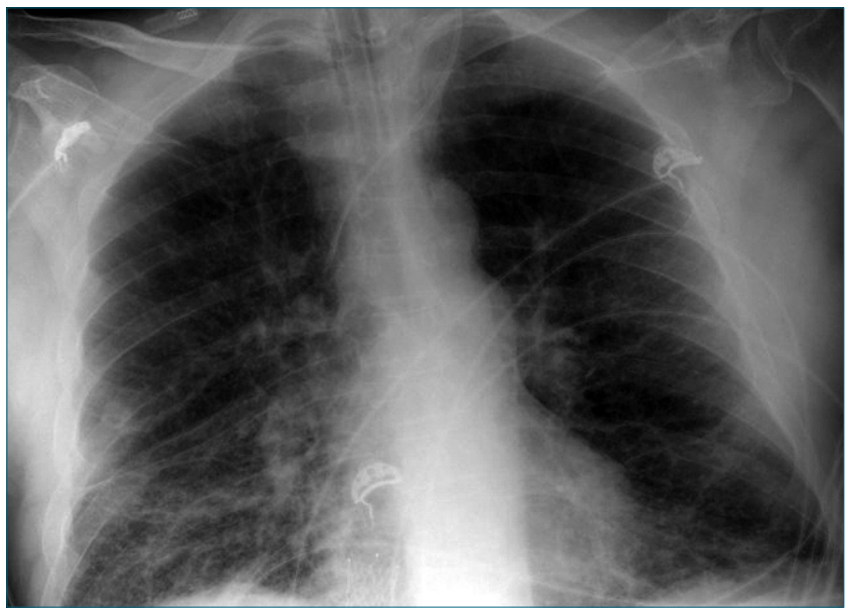

Figura 3. Paciente en ventilación mecánica con catéter yugular izquierdo.

en ventilación mecánica, presentan disfunción del ventrículo derecho. Refiere que aquellos pacientes con neumonía grave como causa de intubación, $\mathrm{PaO}_{2} / \mathrm{FiO}_{2}$ menor de $150, \mathrm{PaCO}_{2}$ alta y mayor presión traspulmonar, presentan mayor probabilidad de fallo de ventrículo derecho si reúnen dos de estas características. En los pacientes COVID-19 (ingresados por insuficiencia respiratoria, en ventilación mecánica) los estudios sugieren que hasta el $40 \%$ pueden presentar este fallo[13].

Debido a la hipoxemia, nuestro paciente precisó tres sesiones de pronación, relajación neuromuscular y óxido nítrico (por hipoxemia y tras retirada de antihipertensivos pulmonares). Durante este año, múltiples revisiones y estudios han demostrado que la relajación y pronación en los pacientes con SDRA, facilitan la oxigenación y ayudan a realizar ventilación protectora[14],[15]. Nuestro paciente, durante los primeros días de ingreso, asoció insuficiencia renal aguda con hiperpotasemia, sin precisar terapia renal sustitutiva. En un interesante artículo publicado en la Revista Chilena de Anestesia, refiere que el fallo renal en el paciente COVID-19 en ventilación mecánica aumenta la mortalidad, relacionándose con valores de ferritina alta, gravedad del paciente y asociación con otros fallos orgáni$\cos [16]$.

Durante la evolución de nuestro paciente, se objetivó pico febril con aumento de reactantes de fase aguda, leucocitosis y empeoramiento del infiltrado radiológico pulmonar. Se inició cobertura antibiótica empírica con meropenem y linezolid y se solicitaron cultivos, realizándose lavado bronquioalveolar. Cabe destacar que, pese a que los pacientes ingresados por neumonía COVID-19 no suelen presentar coinfección bacteriana a su ingreso, estudios ponen de manifiesto que alrededor del $50 \%$ desarrollan neumonía nosocomial. Estas cifras son mayores que las neumonías causadas por virus influenza u otras neumonías bacterianas que requieren ventilación mecánica. Cerca del 80\% de estos aislamientos corresponden a bacilos gram negativos. [17]. Finalmente, en los resultados de los cultivos se objetivó un enterococo en urocultivo y el broncoaspirado fue positivo para Aspergillus (Figura 4).

En un estudio publicado en 2009 donde se recogen 69 autopsias realizadas en pacientes con SDRA, se objetiva que

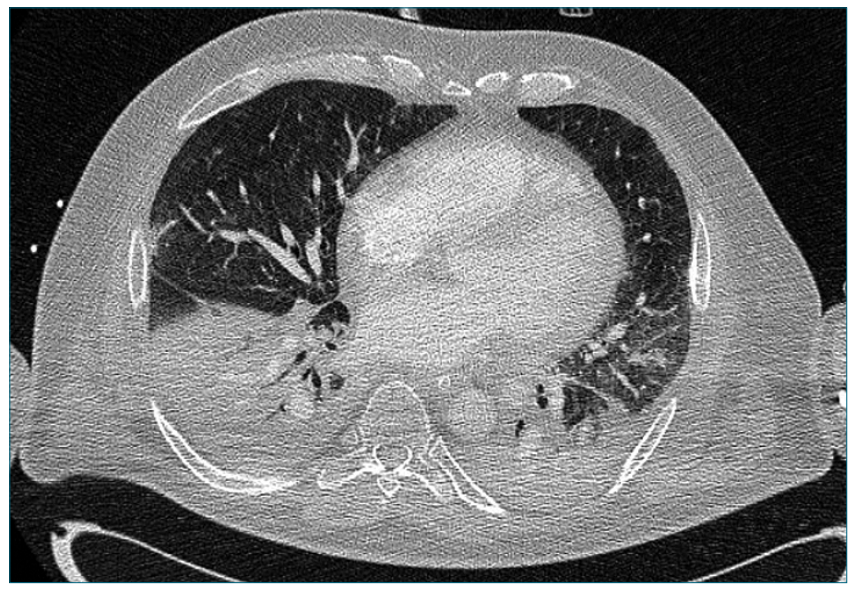

Figura 4. Imagen de corte TAC realizado al paciente tras diagnóstico de aspergilosis.

8 pacientes $(12,5 \%)$ presentaban aspergilosis pulmonar invasiva[18]. Dado que en el paciente con SDRA por coronavirus puede ser incluso mayor, recomendamos realizar la búsqueda sistemática de este microorganismo. Tratamos la asperigilosis con isavuconazol. El isavuconazol es un antifúngico de cobertura idéntica al voriconazol con la salvedad de que produce menor daño gastrointestinal y alteraciones hepatobiliares[19],[20]. Siendo nuestro paciente un paciente trasplantado hepático, optamos por este fármaco.

El 12 de febrero de 2021, se realizó una nueva PCR en exudado nasofaríngeo siendo esta negativa. Los niveles de anticuerpos lgG, eran más de $40.000 \mathrm{AU} / \mathrm{ml}$. En el momento actual, la carga viral de SARS-CoV-2 no se asocia a mayor gravedad de la enfermedad[21]. Sin embargo, los pacientes más graves, sí presentan un número de anticuerpos más altos que aquellos menos graves[22]. Esto se puede deber a un daño potencial producido por una respuesta de anticuerpos subóptima[23].

En el día 15 de ingreso, se realiza traqueotomía percutánea en la unidad de cuidados intensivos sin incidencias. El tiempo de realización de la traqueotomía sigue siendo un tema de debate. Recientemente, se han publicado numerosos estudios de traqueotomía en distrés. La traqueotomía percutánea intraUCl, no presenta mayores complicaciones que la quirúrgica y su coste es menor[25]. Un metaanálisis publicado en la revista JAMA refiere que su realización en los primeros siete días puede estar asociado a menor incidencia de infección nosocomial y días de ventilación mecánica sin mayor mortalidad[26] (Figura 5).

Tras realización de traqueotomía y mejoría de la oxigenación, se comienza a retirar sedación. Progresivamente, el paciente va despertando y se comienza la rehabilitación. Se sienta en el sillón por primera vez a las tres semanas de ingreso. La recuperación de la movilidad no sólo se asocia con mejora de supervivencia intra- $\mathrm{UCl}$, sino incluso con menor tasa de reingresos en función de la capacidad alcanzada[27]. Además, se optimiza balance con pérdida de aproximadamente un litro por día desde el día 24 al 37 de ingreso. Finalmente, el paciente es decanulado el 5 de marzo de 2021 y dado de alta dos días después. 


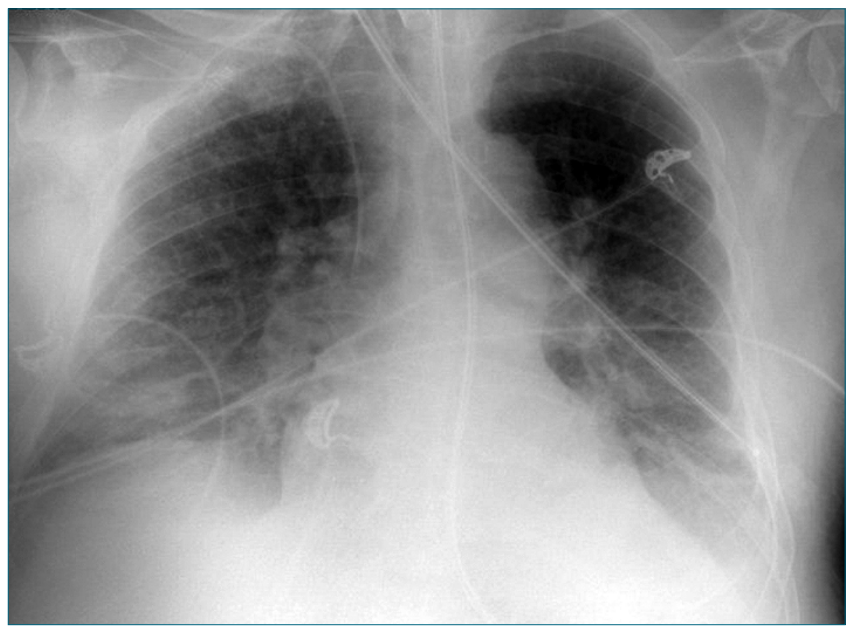

Figura 5. Radiografía de tórax control traqueotomía percutánea.

\section{Referencias}

1. Cyranoski $D$. The biggest mystery: what it will take to trace the coronavirus source. Nature. 2020 Jun 5. doi: 10.1038/d41586020-01541-z. Epub ahead of print. PMID: 32504020.

2. Wu Z, McGoogan JM. Characteristics of and Important Lessons From the Coronavirus Disease 2019 (COVID-19) Outbreak in China: Summary of a Report of 72314 Cases From the Chinese Center for Disease Control and Prevention. JAMA. 2020 Apr 7;323(13):1239-1242. doi: 10.1001/jama.2020.2648. PMID: 32091533.

3. Fine $P$, Eames K, Heymann DL. "Herd immunity": a rough guide. Clin Infect Dis. 2011 Apr 1;52(7):911-6. doi: 10.1093/cid/cir007. PMID: 21427399.

4. Wiersinga WJ, Rhodes A, Cheng AC, Peacock SJ, Prescott HC. Pathophysiology, Transmission, Diagnosis, and Treatment of Coronavirus Disease 2019 (COVID-19): A Review. JAMA. 2020 Aug 25;324(8):782-793. doi: 10.1001/jama.2020.12839. PMID: 32648899.

5. Ricard, JD., Roca, O., Lemiale, V. et al. Use of nasal high flow oxygen during acute respiratory failure. Intensive Care Med 46, 2238-2247 (2020).

6. RECOVERY Collaborative Group, Horby P et al. Dexamethasone in Hospitalized Patients with COVID-19. N Engl J Med. 2021 Feb 25;384(8):693-704. doi: 10.1056/NEJMoa2021436. Epub 2020 Jul 17. PMID: 32678530; PMCID: PMC7383595.

7. Tomazini BM, et al. LCP; COALITION COVID-19 Brazil III Investigators. Effect of Dexamethasone on Days Alive and Ventilator-Free in Patients With Moderate or Severe Acute Respiratory Distress Syndrome and COVID-19: The CODEX Randomized Clinical Trial. JAMA. 2020 Oct 6;324(13):1307-1316. doi: 10.1001/ jama.2020.17021. PMID: 32876695; PMCID: PMC7489411.

8. Arabi YM, Chrousos GP, Meduri GU. The ten reasons why corticosteroid therapy reduces mortality in severe COVID-19. Intensive Care Med. 2020 Nov;46(11):2067-2070. doi: 10.1007/s00134020-06223-y. Epub 2020 Oct 7. PMID: 33026460; PMCID: PMC7538533.

9. Biran $\mathrm{N}$, et al. Tocilizumab among patients with COVID-19 in the intensive care unit: a multicentre observational study. Lancet
Rheumatol. 2020 Oct;2(10):e603-e612. doi: 10.1016/\$26659913(20)30277-0. Epub 2020 Aug 14. PMID: 32838323; PMCID: PMC7428303.

10. Stone JH, et al. BACC Bay Tocilizumab Trial Investigators. Efficacy of Tocilizumab in Patients Hospitalized with Covid-19. N Engl J Med. 2020 Dec 10;383(24):2333-2344. doi: 10.1056/ NEJMoa2028836. Epub 2020 Oct 21. PMID: 33085857; PMCID: PMC7646626.

11. Soriano MC, Vaquero $C$, Ortiz-Fernández $A$, Caballero $A$, Blandino-Ortiz A, de Pablo R. Low incidence of co-infection, but high incidence of ICU-acquired infections in critically ill patients with COVID-19. J Infect. 2021 Feb;82(2):e20-e21. doi: 10.1016/j. jinf.2020.09.010. Epub 2020 Sep 19. PMID: 32956729; PMCID: PMC7501527.

12. Higuera J, Cabestrero D, Gallego S. Análisis función ventricular derecha, función renal y fluidos tras resucitación en pacientes con ventilación mecánica. Revista Chilena de Anestesia Vol. 48 Núm. 3 pp. 246-253|https://doi.org/10.25237/revchilanestv48n03.08

13. Szekely Y, et al. Spectrum of Cardiac Manifestations in COVID-19: A Systematic Echocardiographic Study. Circulation. 2020 Jul 28;142(4):342-353. doi: 10.1161/CIRCULATIONAHA.120.047971. Epub 2020 May 29. PMID: 32469253; PMCID: PMC7382541.

14. Hraiech S, Yoshida T, Annane D, Duggal A, Fanelli V, Gacouin A, Heunks L, Jaber S, Sottile PD, Papazian L. Myorelaxants in ARDS patients. Intensive Care Med. 2020 Dec;46(12):2357-2372. doi: 10.1007/s00134-020-06297-8. Epub 2020 Nov 7. PMID: 33159530; PMCID: PMC7648542.

15. Guérin C, Albert RK, Beitler J, Gattinoni L, Jaber S, Marini JJ, Munshi L, Papazian L, Pesenti A, Vieillard-Baron A, Mancebo J. Prone position in ARDS patients: why, when, how and for whom. Intensive Care Med. 2020 Dec;46(12):2385-2396. doi: 10.1007/ s00134-020-06306-w. Epub 2020 Nov 10. PMID: 33169218; PMCID: PMC7652705.

16. Gallego S. Higuera J. et al. Acute renal failure in COVID-19 patients on mechanical ventilation. Revista Chilena de Anestesia Vol. 49 Núm. 6 pp. 867-873. https://doi.org/10.25237/revchilanestv49n06-12

17. Rouzé $A$, et al. coVAPid study Group. Relationship between SARS-CoV-2 infection and the incidence of ventilator-associated lower respiratory tract infections: a European multicenter cohort study. Intensive Care Med. 2021 Feb;47(2):188-198. doi: 10.1007/s00134-020-06323-9. Epub 2021 Jan 3. PMID: 33388794; PMCID: PMC7778569.

18. de Hemptinne Q, Remmelink M, Brimioulle S, Salmon I, Vincent JL. ARDS: a clinicopathological confrontation. Chest. 2009 Apr;135(4):944-949. doi: 10.1378/chest.08-1741. Epub 2008 Dec 31. PMID: 19118274

19. Patterson TF, et al. Practice Guidelines for the Diagnosis and Management of Aspergillosis: 2016 Update by the Infectious Diseases Society of America. Clin Infect Dis. 2016 Aug 15;63(4):e1e60. doi: 10.1093/cid/ciw326. Epub 2016 Jun 29. PMID: 27365388; PMCID: PMC4967602.

20. Maertens JA, et al. Isavuconazole versus voriconazole for primary treatment of invasive mould disease caused by Aspergillus and other filamentous fungi (SECURE): a phase 3, randomised-controIled, non-inferiority trial. Lancet. 2016 Feb 20;387(10020):760-9. doi: 10.1016/S0140-6736(15)01159-9. Epub 2015 Dec 10. PMID: 26684607.

21. Jacot D, Greub G, Jaton K, Opota O. Viral load of SARS-CoV-2 
across patients and compared to other respiratory viruses. Microbes Infect. 2020 Nov-Dec;22(10):617-621. doi: 10.1016/j. micinf.2020.08.004. Epub 2020 Sep 7. PMID: 32911086; PMCID: PMC7476607.

22. Zhao J, et al. Antibody Responses to SARS-CoV-2 in Patients With Novel Coronavirus Disease 2019. Clin Infect Dis. 2020 Nov 19;71(16):2027-2034. doi: 10.1093/cid/ciaa344. PMID: $32221519 ;$ PMCID: PMC7184337.

23. Iwasaki A, Yang Y. The potential danger of suboptimal antibody responses in COVID-19. Nat Rev Immunol. 2020 Jun;20(6):339341. doi: 10.1038/s41577-020-0321-6. PMID: 32317716; PMCID: PMC7187142.

24. Arabi YM, Chrousos GP, Meduri GU. The ten reasons why corticosteroid therapy reduces mortality in severe COVID-19. Intensive Care Med. 2020 Nov;46(11):2067-2070. doi: 10.1007/s00134020-06223-y. Epub 2020 Oct 7. PMID: 33026460; PMCID:
PMC7538533.

25. Higuera J, et al. Traqueostomía en cuidados intensivos en pacientes COVID-19. Acta Colombiana de Cuidado Intensivo. 2020 Nov 13. Spanish. doi: 10.1016/j.acci.2020.10.009. Epub ahead of print. PMCID: PMC7664356.

26. Chorath K, Hoang A, Rajasekaran K, Moreira A. Association of Early vs Late Tracheostomy Placement With Pneumonia and Ventilator Days in Critically III Patients: A Meta-analysis. JAMA Otolaryngol Head Neck Surg. 2021 Mar 11:e210025. doi: 10.1001/ jamaoto.2021.0025. Epub ahead of print. PMID: 33704354; PMCID: PMC7953336.

27. Hammer M, Grabitz SD, Teja B, Serrano M, Eikermann M; SICU Optimal Mobilization Team (SOMT). Functional mobility recovery predicts readmission to the surgical intensive care unit. Intensive Care Med. 2020 May;46(5):1054-1056. doi: 10.1007/s00134020-05972-0. Epub 2020 Mar 27. PMID: 32221648. 